\title{
Esporotricose em felinos domésticos (Felis catus domesticus) em Campos dos Goytacazes, RJ ${ }^{\mathbf{1}}$
}

\author{
Adriana J. Almeida ${ }^{2 *}$, Nathália F. Reis ${ }^{2}$, Camila S. Lourenço ${ }^{2}$, Nina Q. Costa², \\ Maria L.A. Bernardino ${ }^{3}$ e Olney Vieira-da-Motta ${ }^{3}$
}

\begin{abstract}
Almeida A.J., Reis N.F., Lourenço C.S., Costa N.Q., Bernardino M.L.A. \& Vieira-da-Motta O. 2018. [Sporotrichosis in domestic felines (Felis catus domesticus) in Campos dos Goytacazes/RJ, Brazil.] Esporotricose em felinos domésticos (Felis catus domesticus) em Campos dos Goytacazes, RJ. Pesquisa Veterinária Brasileira 38(7):1438-1443. Laboratório de Clínica e Cirurgia Animal, Universidade Estadual do Norte Fluminense Darcy Ribeiro, Av. Alberto Lamego 2000, Parque Califórnia, Campos dos Goytacazes, RJ 28013-602, Brazil. E-mail: jardim@uenf.br

Sporotrichosis is a subcutaneous mycosis of subacute to chronic evolution, caused by the dimorphic and geophilic fungus Sporothrix schenckii. The zoonotic transmission has been highlighted, with domestic cats having an important epidemiological role in the disease. This research aimed to diagnose cases of sporotrichosis in domestic cats treated in the city of Campos dos Goytacazes/RJ, as well as to correlate the positive cases with the clinical signs observed in them, as well as risk factors such as sex, castrated animals or not, among other characteristics. One hundred domestic felines were used, with skin lesions suspected of sporotrichosis, which underwent a thorough clinical evaluation and completion of individual files. The lesions were then selected for material collection in sterile swabs and subsequent laboratory analysis, through cytology and fungal culture. Of the 100 animals analyzed, 66 (66\%) were positive for Sporothrix spp., Being 46 (69.6\%) uncastrated males, $15(22.7 \%)$ uncastrated females, $4(6.06 \%)$ females castrated and $1(1.5 \%)$ castrated male, $89.3 \%$ of whom had access to the street. Concerning the lesion topography and clinical status of the animals, $43(65.2 \%)$ had focal lesions and $23(34.8 \%)$ disseminated lesions, where $21(31.8 \%)$ died or were submitted to euthanasia at the option of tutor. With the results of the exams, it was possible to adequately prescribe the therapeutic protocol and follow up of 40 animals $(60.6 \%)$, and in the cases of the cats referred $(7.5 \%)$, the respective veterinarian was informed of the respective diagnosis so that this To choose their therapeutic behavior.
\end{abstract}

INDEX TERMS: Sporotrichosis, cats, Felis catus domesticus, skin disease, zoonoses, mycoses.

RESUMO.- A esporotricose é uma micose subcutânea de evolução subaguda a crônica, causada pelo fungo dimórfico e geofílico Sporothrix schenckii. A transmissão zoonótica vem recebendo destaque, tendo os felinos domésticos um importante papel epidemiológico na doença. Este trabalho pesquisa teve como objetivo diagnosticar casos de esporotricose em felinos domésticos atendidos no município de Campos dos Goytacazes/RJ, bem como correlacionar os casos positivos

\footnotetext{
${ }^{1}$ Recebido em 2 de agosto de 2017.

Aceito para publicação em 15 de agosto de 2017.

${ }^{2}$ Laboratório de Clínica e Cirurgia Animal, Universidade Estadual Norte Fluminense Darcy Ribeiro (UENF), Av. Alberto Lamego 2000, Parque Califórnia, Campos dos Goytacazes, RJ 28013-602, Brasil. *Autor para correspondência: jardim@uenf.br, drijardim@yahoo.com.br

${ }^{3}$ Laboratório de Sanidade Animal, Universidade Estadual Norte Fluminense Darcy Ribeiro (UENF), Av. Alberto Lamego 2000, Parque Califórnia, Campos dos Goytacazes, RJ 28013-602.
}

com os sinais clínicos observados nos mesmos, assim como fatores de risco tais quais sexo, animais castrados ou não, dentre outras características. Foram utilizados 100 felinos domésticos, portadores de lesões cutâneas suspeitas de esporotricose, os quais passaram por avaliação clínica minuciosa e preenchimento de fichas individuais. As lesões foram então selecionadas para coleta de material em suabes estéreis e posterior análise laboratorial, através da citologia e cultura fúngica. Dos 100 animais analisados, 66 (66\%) foram positivos para Sporothrix spp., sendo $46(69,6 \%)$ machos não castrados, $15(22,7 \%)$ fêmeas não castradas, $4(6,06 \%)$ fêmeas castradas e $1(1,5 \%)$ macho castrado, os quais $89,3 \%$ tinham acesso à rua. Quanto à topografia lesional e estado clínico dos animais, $43(65,2 \%)$ apresentavam lesões focais e $23(34,8 \%)$ lesões disseminadas, onde 21 (31,8\%) vieram à óbito ou foram submetidos a eutanásia por opção do 
tutor. Com os resultados dos exames foi possível a adequada prescrição do protocolo terapêutico e acompanhamento de 40 animais $(60,6 \%)$, e nos casos dos felinos encaminhados (7,5\%), foi reportado ao médico veterinário responsável o respectivo diagnóstico para que este escolhesse sua conduta terapêutica.

TERMOS DE INDEXAÇÃO: Esporotricose, gatos, Felis catus domesticus, dermatopatia, zoonoses, micoses.

\section{INTRODUÇÃO}

O Sporothrix schenckii é um fungo patogênico complexo que está presente no solo em associação com restos vegetais e em regiões de clima temperado e tropical úmido (Nunes \& Escosteguy 2005). É um fungo saprófita ambiental e cosmopolita que existe na forma micelial em temperaturas ambientes de $25-30^{\circ} \mathrm{C}$ e, como levedura, se desenvolve em temperatura corpórea de $37^{\circ} \mathrm{C}$ (Rippon 1988).

A esporotricose pode acometer diversas espécies de animais e já foi descrita em equinos, cães, felinos, bovinos, suínos, camelos, primatas e no homem. A transmissão da doença é resultante da inoculação direta do fungo por meio de arranhadura e/ou mordedura de animais afetados ou por pequenos traumas durante atividades de lazer ou ocupacionais que tenham relação com floricultura, horticultura e jardinagem (Nobre et al. 2002, Schubach et al. 2006, Caus 2013).

Os gatos têm um importante papel epidemiológico na transmissão e propagação da doença, principalmente os não castrados e de livre acesso à rua (Schubach et al. 2004, Madrid et al. 2011), uma vez que as lesões cutâneas nestes animais contêm uma grande quantidade de células fúngicas infectantes que os distinguem de outras espécies e os caracterizam como notável fonte de infecção (Nobre et al. 2001, Souza et al. 2006, Barros et al. 2010, Madrid et al. 2011).

0 diagnóstico é realizado através de exames presuntivos associados aos complementares como a citologia, exame de cultura micológica, histopatologia, provas sorológicas, testes intradérmicos, inoculação em animais e na reação em cadeia de polimerase (Larsson 2010). No entanto, a cultura fúngica é o método definitivo para o diagnóstico da esporotricose (Thrall 2002).

0 itraconazol é o fármaco de escolha para tratamento de felinos com esporotricose, pois apresenta menos efeitos adversos quando comparado aos demais agentes antifúngicos (Bustamante \& Campos 2001, Schubach et al. 2004, Rosser \& Dunstan 2006, Pereira et al. 2010). 0 uso do medicamento deve estender-se por até 30 dias após a cura clínica (Nobre et al. 2002, Nunes \& Escosteguy 2005).

A presente pesquisa objetivou analisar aspectos relevantes relacionados à doença como sinais clínicos e epidemiologia da esporotricose em felinos, relacionando a positividade dos animais aos dados coletados como sexo, origem do paciente, condições de criação, localização das lesões e demais apresentações clínicas a partir de estatísticas realizadas em Campos dos Goytacazes, Rio de Janeiro.

\section{MATERIAL E MÉTODOS}

Os dados foram obtidos por meio de consultas aos felinos domésticos portadores de lesões em mucosas e/ou cutâneas suspeitas da enfermidade e encaminhados para o Hospital Veterinário da Universidade Estadual do Norte Fluminense Darcy Ribeiro (UENF).
Amostras. Foram avaliados clinicamente 100 felinos portadores de lesões suspeitas, escolhidos por conveniência, de ambos os sexos, sendo preenchida uma ficha de anamnese com questionamentos pertinentes, os quais direcionaram a suspeita clínica. Juntamente a ficha individual, o tutor do animal assinou um termo de aceitação, autorizando o exame clínico, uso dos dados do animal, assim como fotografias e a própria coleta para cultura micológica.

As lesões ulceradas e/ou nódulos cutâneos foram selecionados para a coleta, a qual foi realizada com suabes estéries. As amostras em seguida foram encaminhadas para o Laboratório de Sanidade Animal (LSA) do Centro de Ciências Tecnológicas e Agropecuárias (CCTA) da Universidade Estadual Norte Fluminense Darcy Ribeiro (UENF).

Análise laboratorial. A análise laboratorial baseou-se em dois processos: Citologia e a cultura fúngica. Para a realização da citologia, os suabes contendo material exsudativo foram passados em lâminas estéreis, e posteriormente fixados em fogo, corados pela técnica de Gram, e então levados ao microscópico óptico em aumento de 1000x para a identificação das leveduras.

A cultura fúngica, por sua vez, baseou-se inicialmente no isolamento da forma micelial do fungo, através da inoculação do material coletado pelo suabe em placas de petri contendo ágar Sabouraud Dextrose a 4\% (Himedia ${ }^{\circledR}$ ) acrescido de cloranfenicol $(50 \mathrm{mg} / \mathrm{l}$, Sigma-Aldrich $^{\circledR}$, EUA) e cicloheximida (200mg/l, Sigma-Aldrich ${ }^{\circledR}$, EUA), com incubação a $25-29^{\circ} \mathrm{C}$ por 15 dias. Após a visualização do crescimento micelial, um fragmento da colônia foi retirado e inoculado no ágar YPD (levedura, peptona, dextrose) e incubado a $37^{\circ} \mathrm{C}$ por cerca de sete dias, quando as cepas isoladas na forma micelial foram então convertidas para a morfofisiologia de leveduras, para demonstração do dimorfismo característico do fungo.

As estruturas fúngicas, quando presentes, foram identificadas microscopicamente pela observação das morfologias características de acordo com fragmento das colônias em temperaturas de incubação de 25 a $29^{\circ} \mathrm{C}$ e $37^{\circ} \mathrm{C}$.

\section{RESULTADOS E DISCUSSÃO}

\section{Análise laboratorial}

Após a confecção e análise citológica das lâminas em microscopia, observou-se nos animais positivos, a presença de células leveduriformes, ovais e alongadas, em formato de 'charuto' (Fig.1), como já descrito por Xavier et al. (2004) e Galhardo et al. (2008). Salienta-se que apesar de o cultivo ser considerado o diagnóstico ouro para confirmação da doença, é notória a importância do exame direto como coadjuvante do diagnóstico, visto que se baseia em uma técnica rápida de identificação do fungo em exsudato de gatos, já que nestes existe uma significativa riqueza parasitária, de acordo com o citado por Schubach et al. (2004) e Nuttal et al. (2011).

Quanto à cultura fúngica, considerada padrão ouro para diagnóstico da doença, foram analisados parâmetros macroscópicos e microscópicos de ambos os meios. Observou-se então que em meio ágar Sabouraud Dextrose, aonde o fungo realiza sua conversão da morfofisiologia de levedura para micélio, as colônias são filamentosas, de aspecto membranáceo, cor branca nas bordas e centro escuro (Fig.2A), e microscopicamente, notou-se nesse cultivo isolado, micélios com hifas delgadas, ramificadas, assim como aglomerados de conídeos (Fig.2C), de semelhante forma também descrito por Xavier et al. (2004).

Já no segundo meio, o YPD, onde há conversão da morfofisiologia de micélio para levedura, o padrão macroscópico observado na colônia foi principalmente coloração creme e consistência cremosa (Fig.2B), como já citado por Rippon 
(1988) e a microscopia do cultivo, revelou então células alongadas e leveduriformes, como obsevardas em citologia (Fig.2D).

\section{Aspectos clínicos}

Dos 100 felinos analisados com lesões suspeitas de esporotricose, observou-se positividade para a doença em 66 animais (66\%). Dentre os positivos, $46(69,6 \%)$ eram machos não castrados, $15(22,7 \%)$ eram fêmeas não castradas, quatro fêmeas castradas $(6,06 \%)$ e um macho castrado $(1,5 \%)$, conforme descrito na Figura 3.

Dentro desta casuística, 59 felinos $(89,3 \%)$ tinham acesso à rua, e dentre eles, 15 eram animais resgatados em vias públicas e 44 animais domiciliados, porém, com livre acesso ao peridomicílio. Tais características como predominância sexual, condição de esterilização e acesso à rua também foram

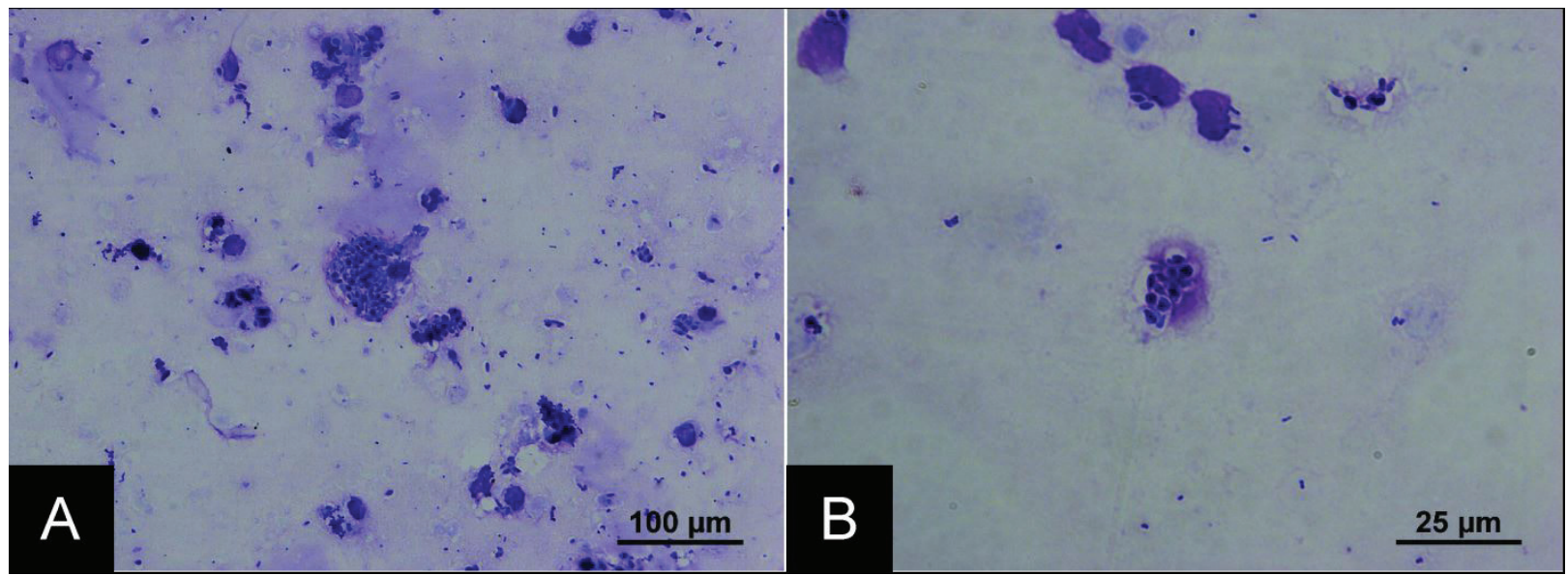

Fig.1. (A, B) Citologia direta das lesões de felinos em Campos dos Goytacazes/RJ, mostrando células leveduriformes e ovaladas semelhantes às de Sporothrix spp. (setas). Método de Gram, obj.100x.
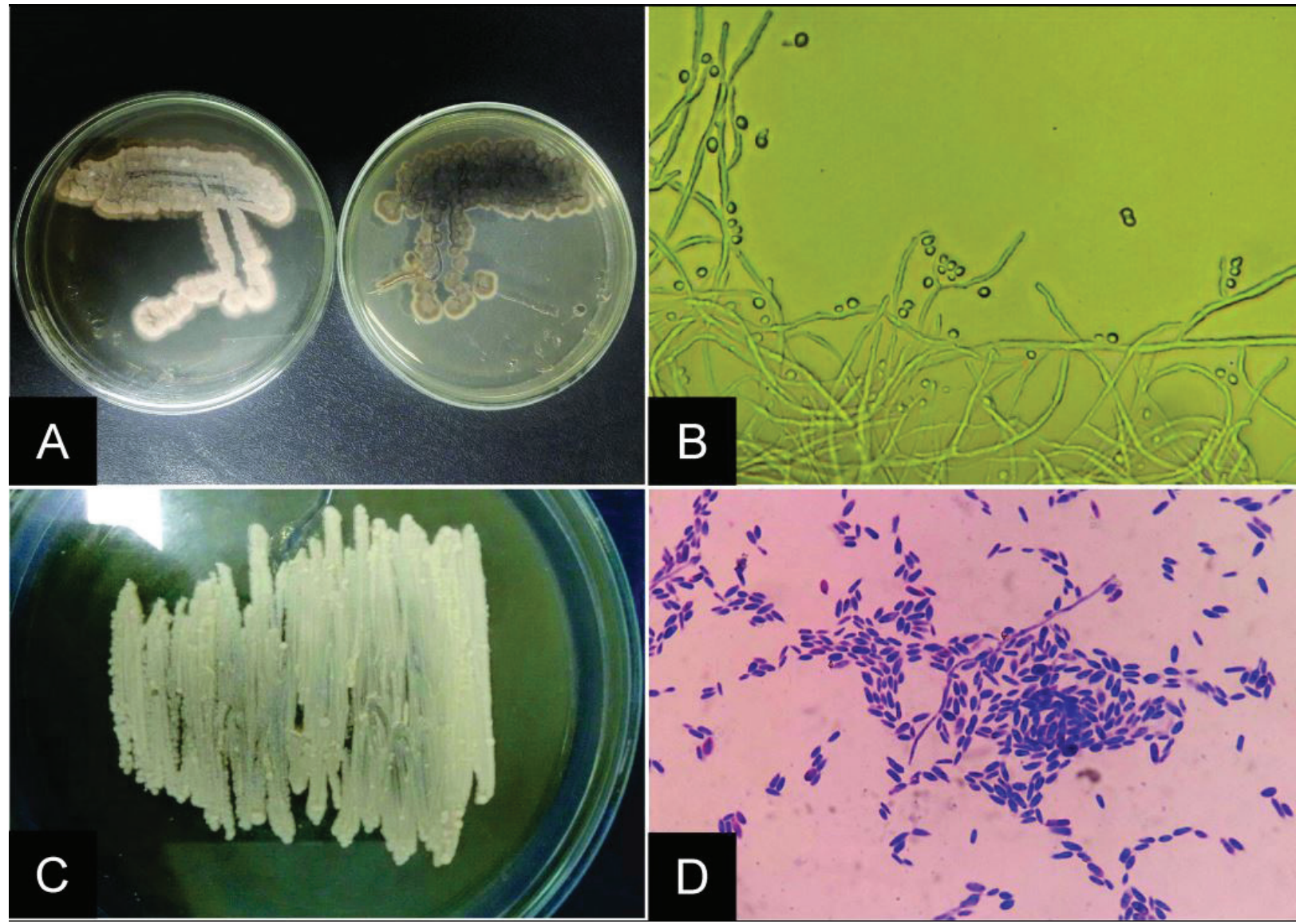

Fig.2. (A) Aspecto macroscópico do fungo Sporothrix sp. isolado em meios ágar Sabouraud Dextrose, (B) em ágar YPD de felinos domésticos, e (C) seu respectivo aspecto microscópico, evidenciando as morfofisiologias de micélio. Método de Gram, obj.40x. (D) Levedura. Método de Gram, obj.100x. Amostras coletadas de felinos domésticos, Campos dos Goytacazes/RJ. 
observadas por Schubach et al. (2001) e Larsson (2000), e ambos atribuíram essa elevada porcentagem de machos a maior permissividade dos tutores à deambulação extradomiciliar, associada aos hábitos característicos da espécie de escavar e encobrir as dejeções com terra, afiação de unhas em materia orgânica, e principalmente mordedura e arranhadura durante disputas territoriais e por fêmeas para acasalamento.

Quanto à distribuição das lesões, 43 felinos (65,2\%) apresentavam lesões focais e $23(34,8 \%)$ lesões disseminadas. Em 26 casos (39,3\%) observou-se comprometimento respiratório, incluindo espirros frequentes e edema de plano nasal (Fig.4).

Gênero e Estado Reprodutivo

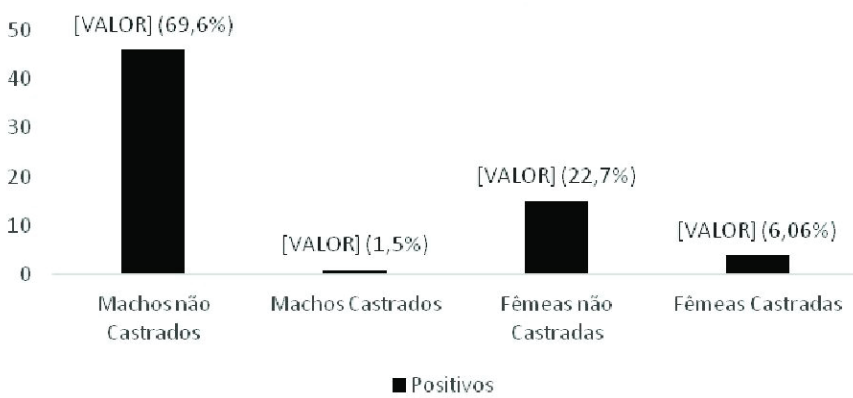

Fig.3. Distribuição de acordo com gênero e estado reprodutivo dos felinos positivos para Sporothrix spp. em Campos dos Goytacazes, RJ.
Conforme estudo realizado por Pereira et al. (2010), a presença de sinais respiratórios é inversamente associada à cura clínica, e diretamente associada a falha terapêutica e ao óbito. A mesma vertente se repetiu neste trabalho de pesquisa, uma vez que animais com acometimento respiratório e sistêmico, somados à inviabilidade de um tratamento intensivo por parte do tutor, tornaram o prognóstico extremamente desfavorável.

Diante de um quadro de infecção disseminada agravada pelo estado imunológico comprometido do animal, observaram-se alterações sistêmicas inespecíficas como a letargia, anorexia, desidratação, levando à taxa de $31,8 \%$ de óbitos ou eutanásias dos animais analisados. De semelhante forma, Chaves (2011) demonstrou em seu estudo sobre a evolução do tratamento clínico de felinos atendidos no Instituto de Pesquisa Clinica Evandro Chagas IPEC/Fiocruz entre 1998 e 2005, o percentual de $23 \%$ casos que evoluíram para óbitos, concluindo então que a freqüência da esporotricose felina é alta, sendo um grande desafio para o acompanhamento dos casos por longo prazo.

Já quanto à topografia lesional, observou-se a predominância de lesões na região cefálica e membros em 47 casos $(71,2 \%)$, conforme as Figuras 5 e 6, visto que são locais mais expostos durante brigas entre os felinos. Salienta-se ainda que, na região cefálica, o local mais acometido foi o plano nasal, seguido de face, orelha e olhos. Contudo, lesões em dorso, região ventral e testicular também foram observadas em 19 casos (28,8\%). Em relação à apresentação dessas lesões, observou-se

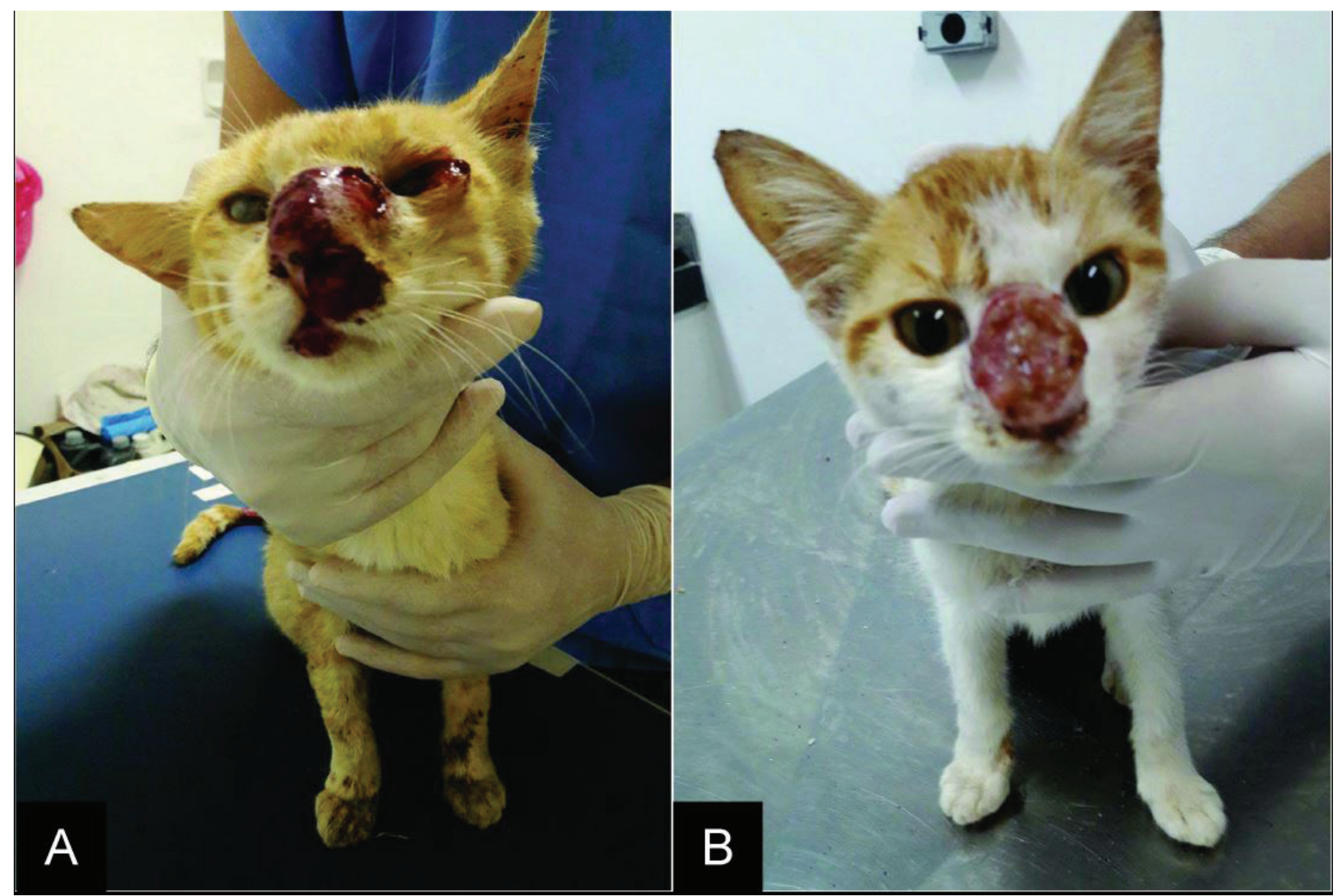

Fig.4. (A, B) Gatas não castradas, resgatadas de vias públicas, com lesões ulceradas em plano nasal, apresentando tumefação na região, dificuldade respiratória e espirros frequentes. 


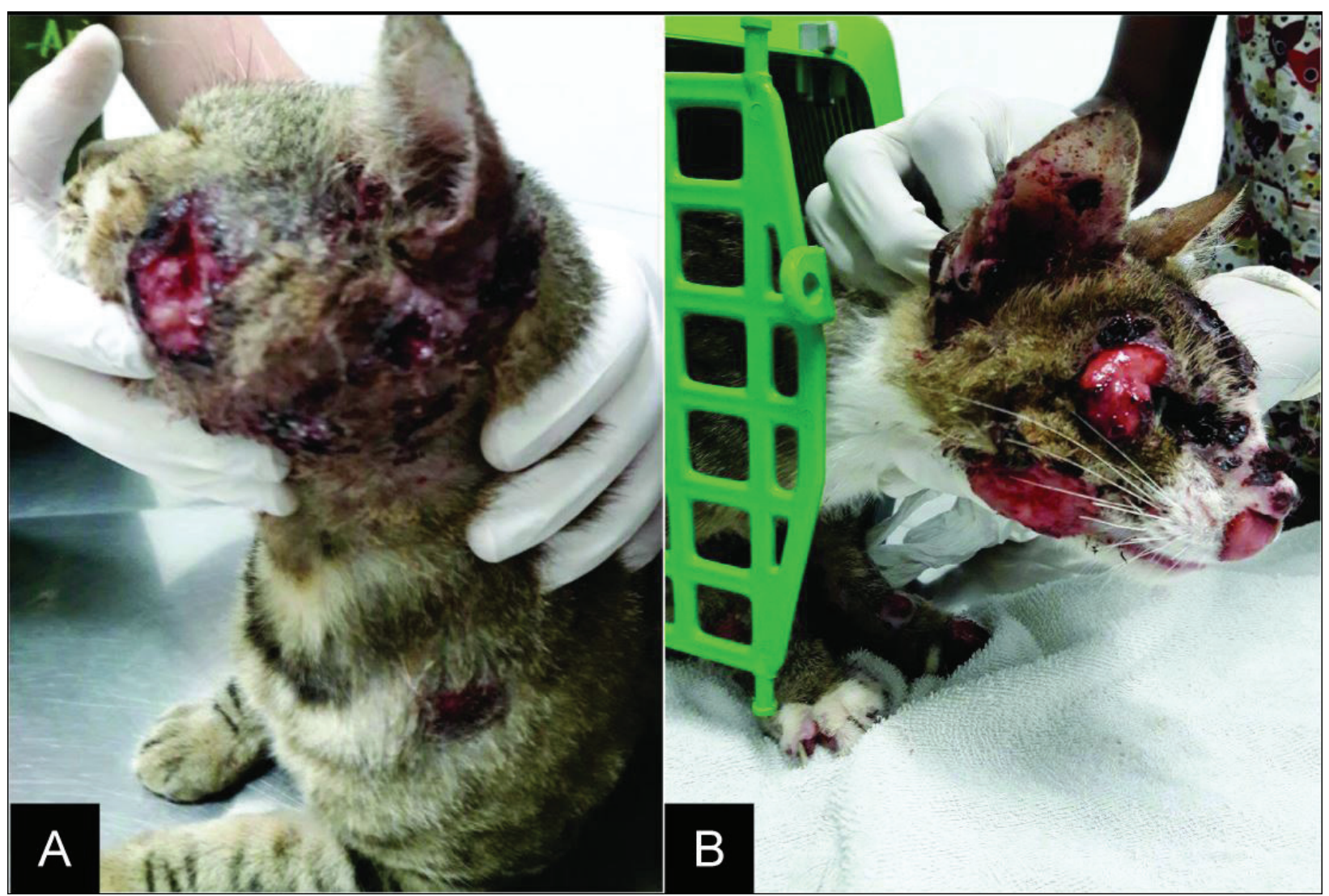

Fig.5. (A, B) Gatos não castrados, resgatados de vias públicas, apresentando múltiplas lesões em face, de aspecto ulcerado e exsudativo.

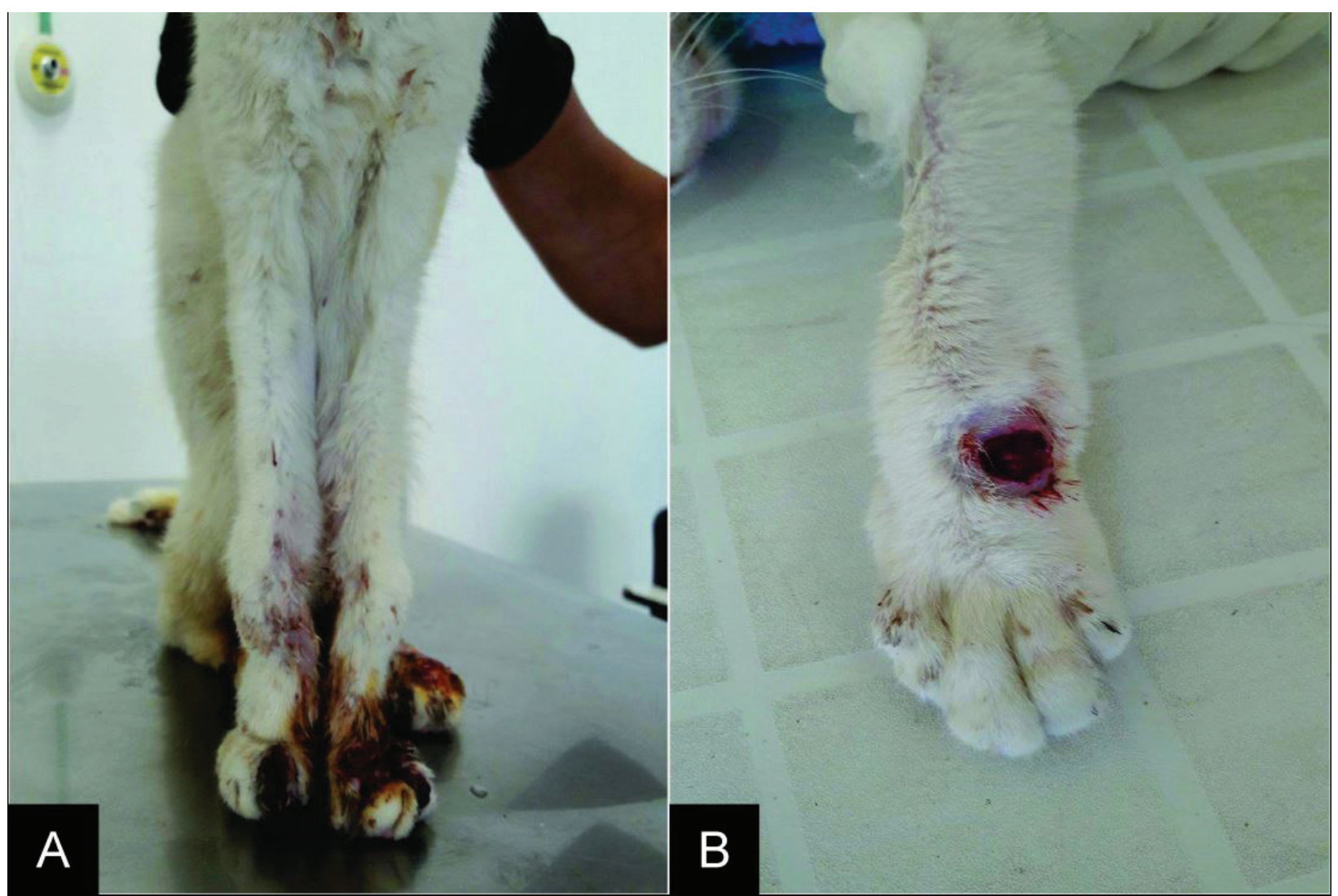

Fig.6. Gatos não castrados, domiciliados, porém, com livre acesso ao peridomicílio. (A) Lesões disseminadas, de caráter ulcerativo em felino apresentando importante caquexia. (B) Felino com lesão circular única em membro anterior esquerdo. 
predominantemente a forma nodular com ulceração central em 47 casos $(71,2 \%)$.

Os resultados positivos foram encaminhados para o tratamento clínico.

\section{CONCLUSÕES}

Houve alta frequência de animais positivos em Campos dos Goytacazes/RJ, sendo a maioria, felinos com livre acesso ao peridomicílio.

Machos não castrados foram os mais acometidos pela esporotricose, seguidos de menor porcentagem de fêmeas não castradas.

As regiões anatômicas mais frequentemente acometidas foram a região cefálica, incluindo face, nariz e pavilhão auricular, além dos membros. Sinais respiratórios, como espirros e edema no plano nasal foram constantemente identificados.

\section{REFERÊNCIAS}

Barros M.B.L., Schubach T.P., Coll J.O., Gremião I.D., Wanke B. \& Schubach A. 2010. Esporotricose: a evolução e os desafios de uma epidemia. Revta. Panam. Salud. Pública 27(6):455-460.

Bustamante B. \& Campos P.E. 2001. Endemic sporotrichosis. Curr. Opin. Infect. Dis. 14(2):145-149. <http://dx.doi.org/10.1097/00001432-20010400000006> <PMid:11979124>

Caus A.L.O. 2013. Esporotricose no estado do espírito santo: um estudo de três décadas. Dissertação de Mestrado, Universidade Federal do Espírito Santo. Disponível em <http://portais4.ufes.br/posgrad/teses/tese_6764_ Dissertac\%26\%23807\%3Ba\%26\%23771\%3Bo\%20Mestrado\%20 Antonio\%20Caus.pdf> Acesso em 1 jul. 2017.

Chaves A.R. 2011. Evolução clínica dos casos de esporotricose felina diagnosticados no Instituto de Pesquisa Clínica Evandro Chagas (IPEC/ Fiocruz) no período de 1998-2005, Rio de Janeiro. Tese de Doutorado em Pesquisa Clínica em Doenças Infecciosas, Instituto de Pesquisa Clínica Evandro Chagas. Disponível em <https://www.arca.fiocruz.br/bitstream/ icict/9310/1/adriana_chaves_ipec_dout_2011.pdf> Acesso em 1 jul. 2017.

Galhardo M.C.G., Oliveira R.M.Z., Valle A.C.F., Paes R.A., Silvatavares P.M., Monzon A., Mellado E., Rodriguez-Tudela J.L. \& Cuenca-Estrella M. 2008. Molecular epidemiology and antifungal susceptibility pattern of Sporothrix schenckii isolates from a cat-transmitted epidemic of sporotrichosis in Rio de Janeiro, Brazil. Med. Mycol. 46(2):141-151. <http://dx.doi. org/10.1080/13693780701742399><PMid:18324493>

Larsson C.E. 2000. Esporotricose. Anais Simpósio Brasileiro sobre Micoses Animais, UFRGS, Porto Alegre, p.66-71. (Resumo)

Larsson C.E. 2010. Esporotricosis, p.433-440. In: Gomez N. \& Guida N. (Eds), Enfermedades Infecciosas em Caninos y Felinos. Intermedica, Buenes Aires.

Madrid I.M., Mattei A.S., Fernandes C.G., Nobre M.O. \& Meireles M.C.A. 2011. Epidemiological findings and laboratory evaluation of sporotrichosis: a description of 103 cases in cats and dogs in southern Brazil. Mycopathologia
173(4):265-273. Disponível em <https://www.ncbi.nlm.nih.gov/ pubmed/22143899> Acesso em 1 jul. 2017.

Nobre M.O., Castro A.P., Caetano D., Souza L.L., Meireles M.C.A. \& Ferreiro L. 2001. Recurrence of sporotrichosis in cats with zoonotic involvement. Revta Iberoam. Micologia 18:137-140.

Nobre M.O., Meireles M.C., Caetano D.T., Faé F., Cordero M., Meireles R.M., Appelt C. \& Ferreiro L. 2002. Esporotricose zoonótica na região sul do Rio Grande do Sul: revisão da literatura brasileira. Revta Bras. Med. Vet. 9(1):36-44.

Nunes F.C. \& Escosteguy C.C. 2005. Esporotricose humana associada à transmissão por gato doméstico. Clínica Veterinária, São Paulo 54:66-68.

Nuttal T., Harvey R.G. \& McKeever P.J. 2011. Manual Colorido de Dermatologia em Cães e Gatos. $2^{\text {a }}$ ed. Revinter Ltda, Rio de Janeiro. 336p.

Pereira S.A., Passos S.R., Silva J.N., Gremião I.D., Figueiredo F.B., Teixeira J.L., Monteiro P.C. \& Schubach T.M. 2010. Response to azolic antifungal agents for treating feline sporotrichosis. Vet. Rec. 166(10):290-294. <http:// dx.doi.org/10.1136/vr.166.10.290><PMid:20208075>

Rippon J. 1988. Sporotrichosis, p.325-352. In: Rippon J. (Ed.), Medical Mycology: the pathogenic fungi and the pathogenic actinomycetes. $3 \mathrm{rd}$ ed. W.B. Saunders, Philadelphia.

Rosser E. \& Dunstan R. 2006. Sporotrichosis, p.645-650. In: Greene C.E (Ed.), Infectious Diseases of the Dog and Cat. 3rd ed. Saunders Elsevier, Philadelphia.

Schubach T.M.P., Valle A.C.F., Gutierrez-Galhardo M.C., Monteiro P.C.F., Reis R.S., Zancopé-Oliveira R.M., Marzochi K.B.F. \& Schubach A. 2001. Isolation of Sporothrix schenckii from the nails of domestic cats (Felis catus). Med. Mycol. 39(1):147-149. <http://dx.doi.org/10.1080/mmy.39.1.147.149> <PMid:11270404>

Schubach T.M., Schubach A., Okamoto T., Barros M.B., Figueiredo F.B., Cuzzi T., Pereira S.A., Santos I.B., Paes R.A., Leme L.R.P. \& Wanke B. 2006. Canine sporotrichosis in Rio de Janeiro, Brazil: clinical presentation, laboratory diagnosis and therapeutic response in 44 cases (1998-2003). Med Mycol. 44(1):87-92.<http://dx.doi.org/10.1080/13693780500148186> $<$ PMid:16805098>

Schubach T.M.P., Schubach A.O., Okamoto T., Figueiredo F.B., Pereira S.A., Leme L.R.P., Santos I.B., Reis R.S., Paes R.A., Perez M.A., Marzochi M.C.A., Francesconi-do-Valle A.C. \& Wanke B. 2004. Utilidade do coágulo sangüíneo para o isolamento de Sporothrix schenckii de gatos naturalmente infectados. Braz. J. Vet. Res. Anim. Sci. 41(6):404-408. <http://dx.doi.org/10.1590/ S1413-95962004000600008>

Souza L.L., Nascente P.S., Nobre M.O., Meinerz A.R.M. \& Meireles M.C.A. 2006. Isolation of Sporothrix schenckii from the nails of healthy cats. Braz. J. Microbiol. 37(3):372-374. <http://dx.doi.org/10.1590/S151783822006000300031>

Thrall M.A. 2002. Cytologic features of head and neck lesions. Proceedings Western Veterinary Conference, California, (Resumo)

Xavier M.O., Nobre M.O., Sampaio Jr D.P., Antunes T.A., Nascente P.S., Sória F.B.A. \& Meireles M.C.A. 2004. Esporotricose felina com envolvimento humano na cidade de Pelotas, RS, Brasil. Ciência Rural 34(6):1961-1963. <http://dx.doi.org/10.1590/S0103-84782004000600047> 